\title{
26668
}

\section{Field Applicability of the g11n Configuration}

\section{S. Szalai* (Hungarian Academy of Sciences), V. Wesztergom (MTA CSFK GGI), K. Szokoli (MTA CSFK GGI), A. Frigy (West-Hungarian University) \& E. Prácser (MTA CSFK GGI)}

\section{SUMMARY}

Firstly a short introduction is given about the theory of the g11n configurations which belong to the group of the geoelectrical quasi null arrays. On the basis of our numerical investigations the application of these arrays seem to be rather perspective. Their field application is however difficult because of the complexity of the inversion of the data of these arrays. These arrays have very large geometric factor, they may change their sign and they can produce very sharp changes. Using an own developed code we inverted field data. The very first results show the field applicability of these configurations. 


\section{Introduction}

Among others due to their difficult applicability geolectric null- (Fig. 1), and quasi null arrays have not been introduced into the practice in spite of that they have been studied for a long time. The application of so-called geometrical null arrays (Szalai et al., 2002) is however not as difficult. On the basis of the research of these arrays the MAN-, and the $\gamma$ quasi null arrays (Fig. 2) were introduced by Szalai et al. (2004) which are applicable in 2D research, too. They have shown that the MAN array may produce higher anomalies than the traditional geoelectric arrays especially when the inhomogeneity is in large depth. The applicability of these arrays, too, has been verified in field by Szalai et al. (2004) in spite of the significant fear of the effect of the noises. In the last years several numerical and field studies have been carried out by the MAN array and its quasi null array versions the so-called $\gamma$ quasi null arrays, which are between the null and traditional arrays according to their theory. The first inversions have been made by the EarthImager, but it was only able to invert data obtained over small-effect inhomogeneities. For such anomalies the results were remarkable (Fig. 3). These arrays may however produce data with negative signs and due to their very/extremely large geometrical factor the resistance values have to be obtained and inverted. Using an own-developed code (Prácser, 2007) we could invert such data. In the presentation the very first results are shown.

\section{Theory}

From the about 100 geoelectric arrays which have ever been used almost the one forth, 25 are null arrays that is arrays which give zero value over a homogeneous half-space (Fig. 1). Most of them however belong to groups III-VII (Fig. 1) following the classification of the arrays by Szalai et al. (2008) that is they are the superposed or focussed arrays whose field application is rather difficult. Szalai et al. (2002) introduced therefore geometrical null arrays where the appropriate positioning of the electrodes results in null array situation. The Schlumberger null- ( $\mathrm{Nr} 23$. in Fig. 1), the threeelectrode null- ( $\mathrm{Nr} 24$. in Fig. 1), and the dipole axial null ( $\mathrm{Nr} 30$. in Fig. 1) arrays were tested on a parallel profile to a quarry wall to detect fractures in limestone (Szalai et al., 2002). Although the test was successful we decided to study null arrays which are linear and applicable also in 2D situations.
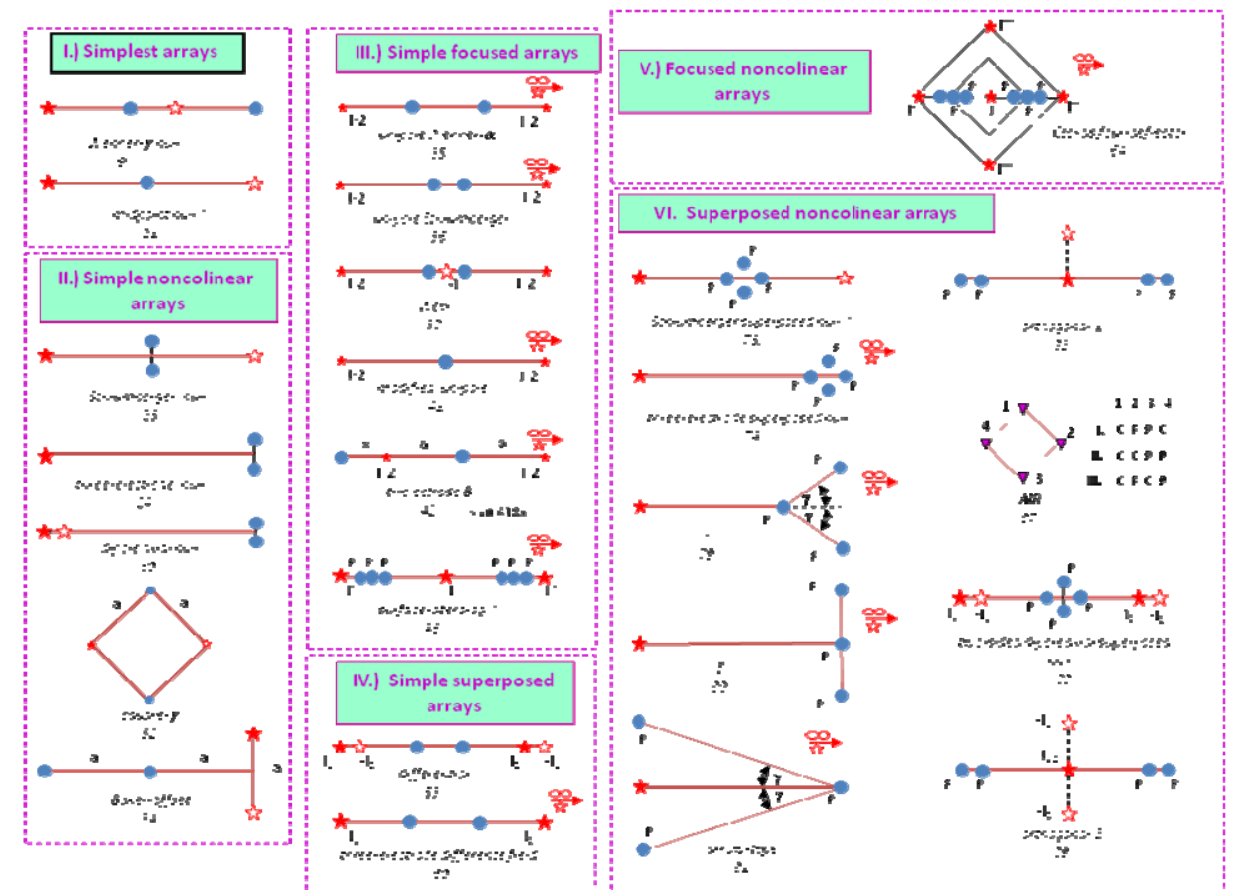

Figure 1 The ever used geoelectric null arrays (from Szalai and Szarka, 2011). Source/sink electrodes are full/empty stars. P: potential electrode (full circles). The lower-case letters such as e, $p, c$ indicate electrodes at infinity. 
There are only two linear null arrays which have symmetry features: the MAN, or midpoint null-, or $\gamma_{11 \mathrm{n}}$ array (Nr 21. in Fig. 1 and the last array in Fig. 2c if $\mathrm{n}=$ inf, Tarkhov, 1957) and the $\gamma$ quasi null array (Fig. 2b). It was shown by Szalai et al. (2004) that the MAN array presents larger anomalies than the Wenner array if the inhomogeneity is in large depth (Fig. 3). They have also shown that null arrays produce well detectable anomalies also in the field even without taking any special attention to the precise positioning of the electrodes.

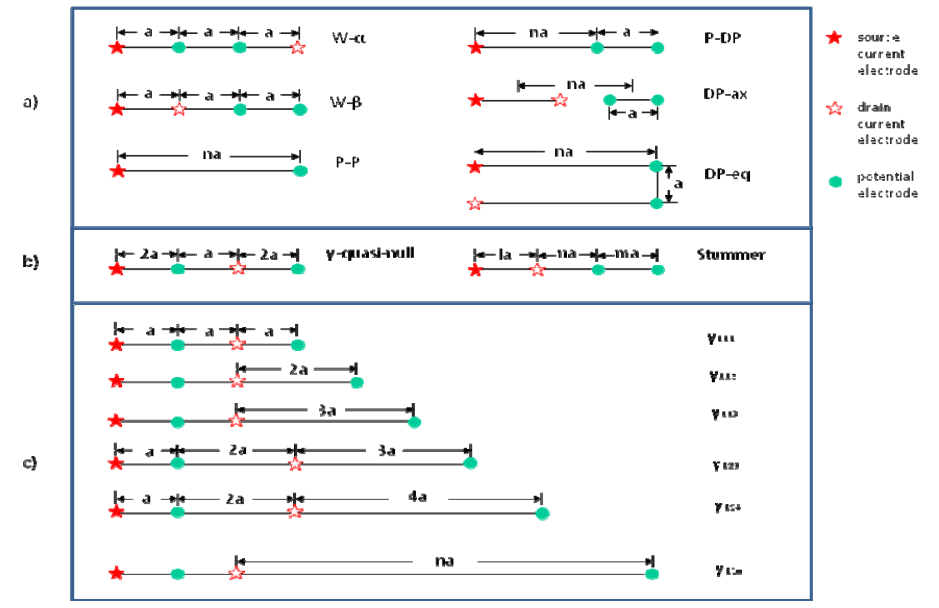

Figure 2 a) Traditional arrays b) $\gamma$ quasi null and Stummer arrays. c) $\gamma 11$ arrays. If $n=$ inf. the $\gamma 11 n$ array turns into the MAN array. Stars denote current electrodes, circles denote potential electrodes.

We studied the possibility of building in such arrays in multielectrode systems. For this aim only the MAN array can be used because of the demand of the equidistance of the electrodes. Studies of this array motivated us however to investigate rather arrays which are similar to the MAN array but neither of their electrodes is in the infinity. The infinite electrode lead namely to serious problems both in the practice and in the numerical modelling. In the present stage of the research we study therefore the so-called $\gamma_{11 \text { n }}$ arrays (Fig. 2c) which are between a traditional array, the $\gamma_{111}$ one (the well-known Wenner- $\gamma$ array) and the MAN array. These arrays represent therefore a transition from a conventional array to a null array. Beside of the theoretical interest of this transition it results in different images supposed to have different drawbacks but also advantages (see e.g. Fig. 4).
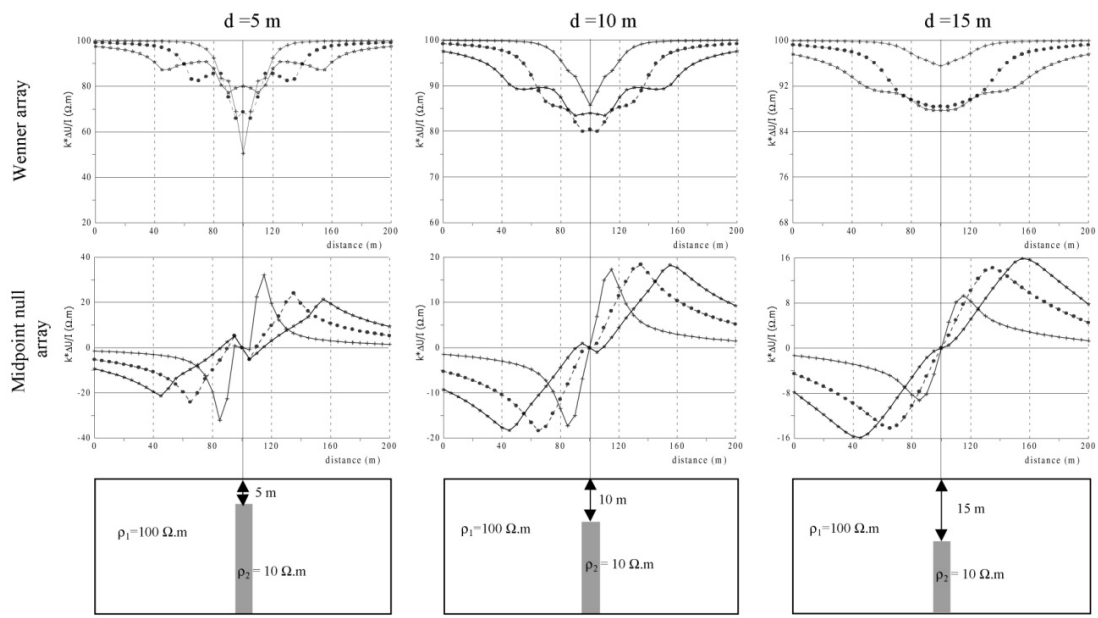

Figure 3 Numerical modeling results over a $2 D$ dyke. $k$ is geometrical coefficient of the Wenner array. The characteristic array lengths are $20(+), 40(\bullet)$ and $60 \mathrm{~m}(*)$.

Acquisition of resistance data is required for these configurations because these arrays may have negative signals and because infinitely large apparent resistivity values may occur. The EarthImager software proved to be appropriate to handle such data if they were generated by the program itself and if the effect of the inhomogeneity was not large. Figure 3 presents images which has been created by 


\section{EAGE}

these arrays and by the Wenner- $\alpha$ and the optimised traditional array, the Stummer array (from Szalai et al. 2015). In many examples - like also in the here presented one - the $\gamma$ arrays, or many of them produced better results than even the optimised traditional array. Unfortunately not even this software could handle our field data therefore the ERT2DInv code was developed (Prácser, 2007). It uses FEM method and linearized smoothed inversion algorithm.

The very first field measurements we carried out over a tube close to the surface (Fig. 4). It was supposed that it can be well seen by all configurations and that the traditional configurations will produce better results for these parameters. Our expectations have been fulfilled. (For the configuration of the traditional arrays see Fig. 2a) We studied also the $\gamma$-quasi null array (Fig. 2b) which is the modified version of the Wenner $\gamma$-null array. With a small modification of the interelectrode distances we got an array close to the null array but it can be build in ERT systems. In its image the corners of the section are strongly distorted. The anomalies of the traditional arrays are remarkable as it was expected. We think however that if such a body would be in larger deepth the $\gamma$ arrays would be more useful. To verify this statement however one should find appropriate test site.

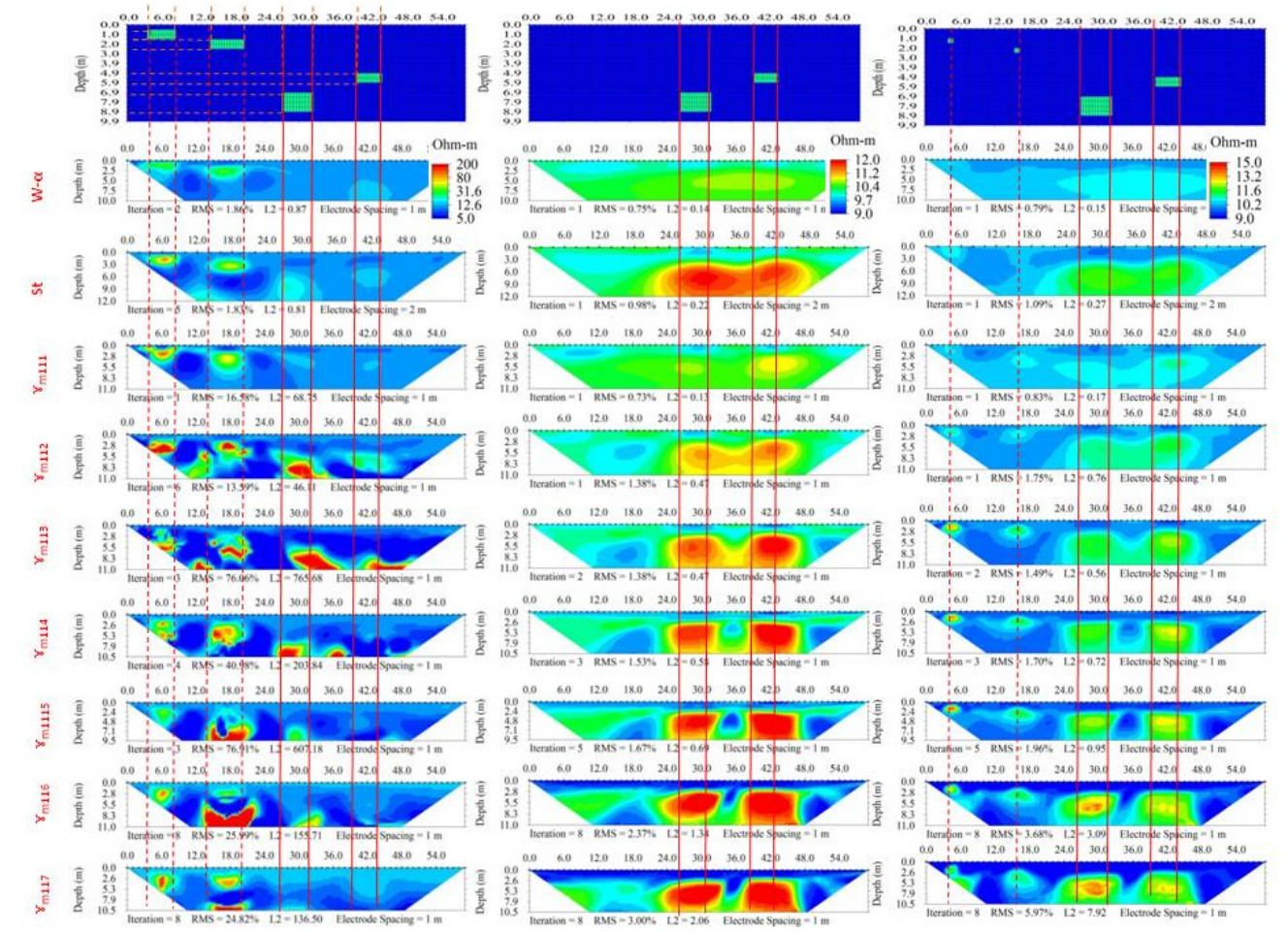

Figure 4 Left column: Inversion results from the Wenner- $\alpha$, Stummer and rm11n (n=1-7) configurations for the model similar to that in Wilkinson et al. (2006). Middle column: results for the same model without the near-surface anomalous bodies. Right column: the first model with smaller near-surface inhomogeneities. The models are given in the first row. 


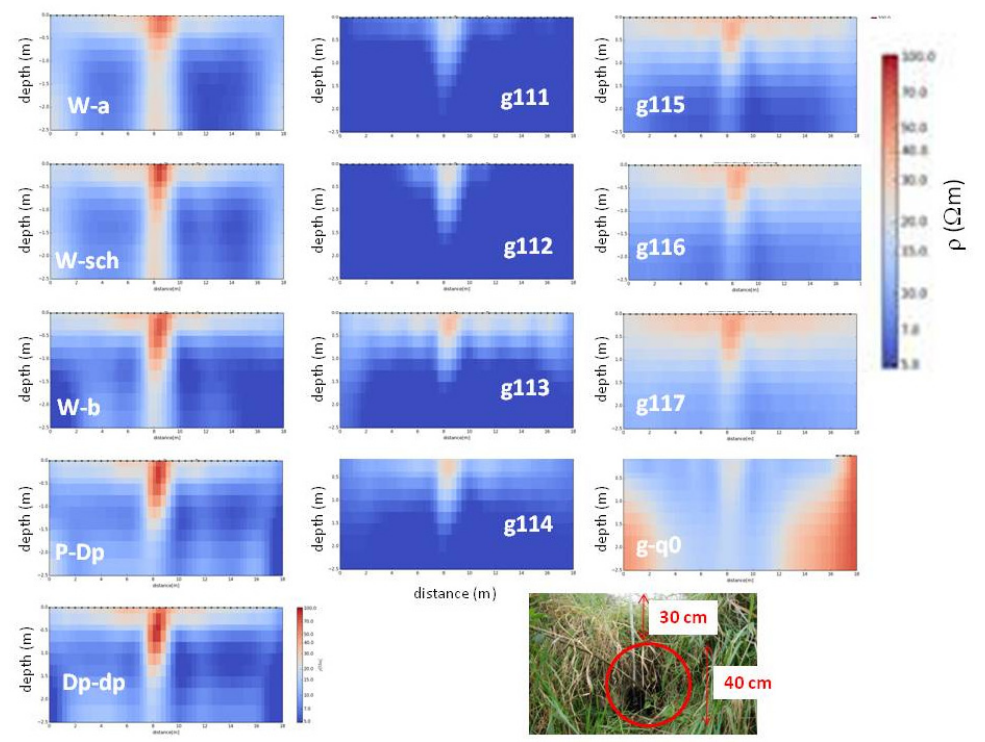

Figure 5 The first inverted $\gamma_{11 n}$ array field data over a tube. The profile was perpendicular to it. Below the image of the tube with its parameters.

\section{Conclusions}

Although geoelectrical null-, and quasi arrays have been studied since very long time they have now been introduced in the practice. We give a short introduction into the theory of the $\gamma$ arrays and show the very first inverted field data results. These results are promising. Now appropriate test sites are looked for.

\section{References}

Falco, P., Negro, F., Szalai, S., Milnes, E. [2013] Fracture characterisation using geoelectric nullarrays. Journal of Applied Geophysics, 93, 33-42.

Szalai, S., Kis, Á., Metwaly, M., Lemperger, I. and Szokoli, K. [2015] Increasing the effectiveness of electrical resistivity tomography using $\gamma 11 \mathrm{n}$ configurations. Geophysical Prospecting, accepted for publication

Szalai, S., Koppán, A, Szokoli, K., Szarka, L. [2013] Geoelectric imaging properties of traditional arrays and of the optimised Stummer configuration. Near Surface Geophysics, 11, 51-62.

Szalai, S., Szarka, L. [2008] On the classification of surface geoelectric arrays. Geophysical Prospecting, 56, 159-175.

Szalai, S., Szarka, L. [2011] Expanding the possibilities of two-dimensional multielectrode systems, with consideration to earlier geoelectric arrays. Journal of Applied Geophysics, 75, 1-8.

Szalai, S., Szarka, L., Marquis, G., Sailhac, P., Kaikkonen, P. and Lahti, I. [2005] Colinear null arrays in geoelectrics. IAGA WG 1.2 on Electromagnetic Induction in the Earth, Proceedings of the $17^{\text {th }}$ Workshop, http://www.emindia2004.org.

Szalai, S., Szarka, L., Prácser, L., Bosch, F., Müller, I. and Turberg, P. [2002] Geoelectric mapping of near-surface karstic fractures by using null arrays. Geophysics, 67, 1769-1778.

Tarkhov, A.G. [1957] On electric geophysical exploration methods of the pure anomaly. Bulletin Izvestia Akademii Nauk USSR, 8, 11-22. 